\title{
Recoil Studies of Photonuclear Reactions at Intermediate Energies
}

\author{
Hiromitsu Haba* \\ Cyclotron Center, RIKEN, Wako, Saitama 351-0198, Japan
}

Received: November 12, 2002; In Final Form: December 16, 2002

A review is given on the recoil studies of photonuclear reactions on complex nuclei at intermediate energies. Recoils of 167 radionuclides formed in the photonuclear reactions of ${ }^{27} \mathrm{Al},{ }^{\text {nat }} \mathrm{V},{ }^{\text {nat }} \mathrm{Cu},{ }^{93} \mathrm{Nb},{ }^{\text {nat }} \mathrm{Ag}$, ${ }^{\text {nat }} \mathrm{Ta}$, and ${ }^{197} \mathrm{Au}$, induced by bremsstrahlung of end-point energies $\left(E_{0}\right)$ from 600 to $1100 \mathrm{MeV}$, have been investigated by the thicktarget thick-catcher method. The recoil velocity from the first step and the mean kinetic energy of the residual nuclei in the second step were deduced based on the two-step vector velocity model and discussed by comparing with the reported results on proton-induced reactions. Recoils of ${ }^{24} \mathrm{Na}$ produced from ${ }^{27} \mathrm{Al},{ }^{\text {nat }} \mathrm{V}$, nat $\mathrm{Cu}$, ${ }^{\text {nat }} \mathrm{Ag}$, and ${ }^{197} \mathrm{Au}$ are of special interest from a viewpoint of a change in the production mechanism with respect to target mass. Reaction yields of 58 and 63 radionuclides produced in the photofission of ${ }^{197} \mathrm{Au}$ and ${ }^{209} \mathrm{Bi}$, respectively, were also measured at $E_{0}=300-1100 \mathrm{MeV}$ by a catcher foil technique with the aid of intensive chemical separation. The fission characteristics such as the charge and mass distributions and the numbers of pre- and post-fission neutrons were determined. These results were compared with the calculations using the Photon-induced Intranuclear Cascade Analysis 3 code combined with the Generalized Evaporation Model code.

\section{Introduction}

Photons are the force carriers of the electromagnetic interaction. The electromagnetic force can act on the scale of the whole nucleus but also on the smaller scale of nucleons, and so this interaction is very useful for probing both global and local properties of the atomic nucleus. At intermediate energies, photons interact with the nucleus through three main processes; giant dipole resonance (GDR), quasi-deuteron mechanism (QDM), and $\Delta$ resonance, which are characteristic for the wavelength of incident photons. These initial interactions of photons with nuclei are purely electromagnetic and are quite different from that of hadron-induced reactions initiated by the strong nucleon-nucleon interaction. It is of interest to investigate whether there exist similarities in the final steps in these two types of nuclear reaction.

By the author's group, yields of bremsstrahlung-induced reactions such as photopion reactions, ${ }^{1-4}$ photospallation, ${ }^{5-8}$ and photofragmentation, ${ }^{9,}$ of various targets from ${ }^{7} \mathrm{Li}$ to ${ }^{209} \mathrm{Bi}$ have been measured radiochemically to extract information on reaction mechanism and nuclear structure. These systematic studies are also of importance for practical applications such as astrophysics, medicine, accelerator technology, and nuclear waste transmutation.

Nuclear recoil experiments give us valuable information such as angular distributions and kinetic energies of product nuclei and deepen our understanding on the reaction mechanism. One of the simplest recoil experiments is done by the thick-target thick-catcher method. It has been extensively applied to studies of hadron-induced reactions on various targets over a wide range of incident particle energy, ${ }^{11-13}$ but rarely to studies of photonuclear reactions. ${ }^{14-17}$ Small cross sections of photonuclear reactions compared with hadroninduced reactions seemed to make the detection of recoil nuclei arduous.

Recently, we have successfully applied the multiple thicktarget thick-catcher method to photospallation reactions to examine the effect due to a difference in the initial interactions

*Corresponding author. E-mail: haba@riken.go.jp. FAX: +81-48467-9777. between photon-induced reaction and hadron-induced one dynamically. ${ }^{18-21}$ The recoil properties of 137 radionuclides formed in the photospallations of ${ }^{27} \mathrm{Al}$, ${ }^{\text {nat }} \mathrm{V},{ }^{\text {nat }} \mathrm{Cu},{ }^{93} \mathrm{Nb}$, ${ }^{\text {nat }} \mathrm{Ag}$, ${ }^{\text {nat }} \mathrm{Ta}$, and ${ }^{197} \mathrm{Au}$, induced by bremsstrahlung of end-point energies $\left(E_{0}\right)$ from 600 to $1100 \mathrm{MeV}$, have been investigated based on the two-step vector velocity model. ${ }^{18-21}$ The recoil properties such as the recoil velocity $v$ from the first step and the kinetic energy $T$ of the residual nuclei in the second step were deduced and discussed by comparing with those of protoninduced reactions. ${ }^{20,21}$

The nuclear recoil experiment by the thick-target thickcatcher method is very useful to obtain fission yields as well as recoil properties, because the energetic fission fragments with small yields can be separated effectively in the catcher foil from the relatively less energetic spallation residues with large yields. Photofissions at intermediate energies are expected to be unique for investigating the complex dynamics of excitation in heavy nuclei, because photons interact electromagnetically with nuclei through GDR, QDM, and $\Delta$ resonance. For preactinide nuclei such as ${ }^{197} \mathrm{Au}$ and ${ }^{209} \mathrm{Bi}$, the photofission processes opened by QDM and $\Delta$ resonance are especially important due to their high fission thresholds $(>20 \mathrm{MeV})$ that suppress the fission channels opened by GDR. Thus a photon sees all nucleons inside a nucleus identically and interacts with a nucleon-nucleon pair or a single nucleon, transferring its kinetic energy effectively but comparatively little angular momentum to the nucleus. The fission process must be regarded as a collective process of the nucleus. It is interesting how these local excitations are propagated in nuclear matter and transferred to the collective nuclear excitation.

Photofission, however, is not very well understood at energies of interest, because fission yields are quite lacking compared with spallation and fragmentation yields. The situation is the same with proton-induced reactions. Recently, Duijvestijn et al. ${ }^{22}$ measured cross sections of proton-induced fissions of ${ }^{\text {nat }} \mathrm{W},{ }^{197} \mathrm{Au},{ }^{\text {nat }} \mathrm{Pb},{ }^{208} \mathrm{~Pb}$, and ${ }^{232} \mathrm{Th}$ at a proton energy of $190 \mathrm{MeV}$. They compared the fission cross sections with theoretical calculations using the intranuclear cascade code LAHET, ${ }^{23}$ and found that the calculation severely underestimates the cross sections for the preactinides and overestimates in general the width of the mass distribution. The absence of a 
reliable theoretical model to predict fission yields as well as the need for experimental yields has motivated us to investigate photofission processes of preactinides. Quite recently, we measured recoil properties and yields of radionuclides produced in the photofission of ${ }^{197} \mathrm{Au}$ and ${ }^{209} \mathrm{Bi}$ at $E_{0}=300-1100$ $\mathrm{MeV}$ by a catcher foil technique with the aid of intensive chemical separation. ${ }^{24-26}$ The recoil properties such as the forward-to-backward ratio $F / B$ and the kinetic energy $T$ of the residual nuclei were discussed by comparing with those of photospallation products and also with the literature data for proton-induced reactions. ${ }^{26}$ Furthermore, fission characteristics such as the charge and mass distributions and the numbers of pre- and post-fission neutrons were determined based on the 58 and 63 fission yields on ${ }^{197} \mathrm{Au}$ and ${ }^{209} \mathrm{Bi}$, respectively. ${ }^{24,26}$

By the author's group, the reaction yields of photopion and photospallation reactions and the kinetic energies of the photospallation products were systematically compared with the Photon-induced Intranuclear Cascade Analysis (PICA) code developed by Gabriel and coworkers, ${ }^{27,} 28$ and various information on reaction mechanism and nuclear structure was extracted. ${ }^{1-4,8,18-21}$ Recently, the original code was modified by $\mathrm{Fu}^{29}$ and Sato et al. ${ }^{30,31}$ and the fission process was considered for the first time in the code based on the Generalized Evaporation Model (GEM) code developed by Furihata. ${ }^{32}$ Comparisons of the experimental results with the latest PICA calculations ${ }^{31}$ are under study in our group. ${ }^{26,33,34}$

In the following, our recoil studies of photonuclear reactions at intermediate energies are reviewed based on the cumulative results in the series of the author's papers. ${ }^{18-21,24-26,33,34}$

\section{Experimental Procedures}

Irradiations by bremsstrahlung beams with end-point energies of $E_{0}=300-1100 \mathrm{MeV}$ were carried out using the 1.3$\mathrm{GeV}$ electron synchrotron of the High Energy Accelerator Research Organization (KEK) at Tanashi. A $50 \mu \mathrm{m}$ thick Pt foil was used as a radiator for bremsstrahlung production, and the beams were collimated to $20 \mathrm{~mm}$ in diameter at the irradiation position. The targets consisted of a stack of 5-50 sets of a target foil of $25 \times 25 \mathrm{~mm}^{2}$ in size sandwiched by one pair of 3.5 or $7.0 \mathrm{mg} \mathrm{cm}^{-2}$ thick Mylar foils of the same size, which collected the recoil nuclei in the forward and backward directions with respect to the beam direction. The target thicknesses were $6.6,14,22,24,32,32,90$, and $900 \mathrm{mg} \mathrm{cm}^{-2}$ for ${ }^{27} \mathrm{Al},{ }^{\text {nat }} \mathrm{V},{ }^{\text {nat }} \mathrm{Cu},{ }^{93} \mathrm{Nb},{ }^{\text {nat }} \mathrm{Ag}$, ${ }^{\text {nat }} \mathrm{Ta},{ }^{197} \mathrm{Au}$, and ${ }^{209} \mathrm{Bi}$, respectively. The photon intensities evaluated from the monitor reaction of ${ }^{27} \mathrm{Al}(\gamma, 2 \mathrm{pn})^{24} \mathrm{Na}$ (Ref. 35) were $10^{9}-10^{10}$ equivalent quanta per second (eq.q. $\mathrm{s}^{-1}$ ). The typical irradiation periods were $3 \mathrm{~h}$ for ${ }^{27} \mathrm{Al},{ }^{\text {nat }} \mathrm{V}$, nat $\mathrm{Cu},{ }^{93} \mathrm{Nb}$, and ${ }^{209} \mathrm{Bi}, 4 \mathrm{~h}$ for ${ }^{\text {nat }} \mathrm{Ag}$ and ${ }^{\text {nat }} \mathrm{Ta}$, and $5 \mathrm{~h}$ for ${ }^{197} \mathrm{Au}$. After irradiation, chemical separation of $\mathrm{K}, \mathrm{Sc}, \mathrm{Fe}$, $\mathrm{Ni}, \mathrm{Zn}, \mathrm{Ga}, \mathrm{As}, \mathrm{Rb}, \mathrm{Sr}, \mathrm{Y}, \mathrm{Zr}, \mathrm{Nb}, \mathrm{Mo}, \mathrm{Ag}, \mathrm{Ba}$, and rare earth elements (REE) from $\mathrm{Au}$, and of $\mathrm{Fe}, \mathrm{Ga}, \mathrm{As}, \mathrm{Br}, \mathrm{Sr}, \mathrm{Y}, \mathrm{Zr}, \mathrm{Nb}$, $\mathrm{Ag}, \mathrm{I}$, and $\mathrm{Ba}$ from $\mathrm{Bi}$ was employed for part of the targets to detect radionuclides with small yields. In addition, the chemical separation of $\mathrm{Sc}, \mathrm{Ba}$, and REE from the Au target and its catchers was performed in the separate irradiations. The details of the chemical procedures are described in References 21,26 , and 36. Some selected target foils and all of the forward and backward catchers from one target pile as well as the chemically separated samples were assayed for radioactivities with Ge detectors.

\section{PICA3/GEM Calculation}

Gabriel and coworkers ${ }^{27,} 28$ developed a Monte-Carlo intranuclear cascade-evaporation analysis code for photonuclear reactions: Photon-induced Intranuclear Cascade Analysis (PICA). This calculational code is based on the Bertini version ${ }^{37}$ of the intranuclear cascade and differs only with respect to the initial interactions. The initial interactions considered are QDM and the single pion photoproduction through $\Delta$ resonance. The code is valid for photon energies from 30 to $400 \mathrm{MeV}$. Recently, $\mathrm{Fu}^{29}$ and Sato et al. ${ }^{30,31}$ modified the original code and named the new one as PICA3. The energy limit was extended to $E_{0}=0-3.5 \mathrm{GeV}$ by considering GDR and multiple-pion production channels. ${ }^{29}, 30$ The angular distribution of nucleons produced by the quasi-deuteron disintegration as well as its cross section was revised..$^{30}$ In addition, the nucleon-nucleon scattering cross section used in the cascade process, the level density parameter and the mass formula used in the evaporation process were updated. ${ }^{30}$ The nuclear medium effect and the nuclear shadowing effect were also considered in the photopion production cross sections. ${ }^{31}$ On the other hand, Furihata ${ }^{32}$ developed the GEM code based on the Generalized Evaporation Model and the Rutherford Appleton Laboratory (RAL) model by Atchison. ${ }^{38}$ Parameters used in the RAL model ${ }^{38}$ which determine the mass, charge, and kinetic energy distributions of fission fragments were reevaluated. Furihata ${ }^{32}$ estimated the cross sections for nuclides produced from the proton-induced reactions of ${ }^{208} \mathrm{~Pb}$ and ${ }^{197} \mathrm{Au}$ using a combination of the intranuclear cascade model (INC) in the LAHET code ${ }^{23}$ and the GEM code. ${ }^{32}$ It was found that the INC/GEM code ${ }^{32}$ reproduces successfully the cross sections for fission fragments as well as light fragments.

Quite recently, Sato et al. ${ }^{31}$ combined the GEM code ${ }^{32}$ with the PICA3 code ${ }^{31}$ to describe the evaporation and fission processes in the photonuclear reactions. In this paper, the reproducibility of the kinetic energies and reaction yields of residual nuclei by the PICA3/GEM $\operatorname{code}^{31}$ was examined systematically. The input parameters recommended in the original papers ${ }^{28,31,32}$ were used in the present calculation.

\section{Results and Discussion}

\subsection{Recoil Properties of Photospallation Products on} Complex Nuclei

4.1.1. Recoil Fractions and Effective Mean Ranges. Radioactivities of $1,14,24,26,31,21$, and 20 nuclides produced from ${ }^{27} \mathrm{Al}$, nat $\mathrm{V}$, ${ }^{\text {nat }} \mathrm{Cu},{ }^{93} \mathrm{Nb}$, ${ }^{\text {nat }} \mathrm{Ag}$, ${ }^{\text {nat }} \mathrm{Ta}$, and ${ }^{197} \mathrm{Au}$, respectively, were identified both in the target and catcher foils. From the fractions of each nuclide measured in the forward and backward catcher foils, expressed as $F=N_{\mathrm{F}} /\left(N_{\mathrm{F}}+N_{\mathrm{B}}\right.$ $\left.+N_{\text {target }}\right)$ and $B=N_{\mathrm{B}} /\left(N_{\mathrm{F}}+N_{\mathrm{B}}+N_{\text {target }}\right), N$ being the number of atoms, the effective mean ranges $F W$ and $B W$ were obtained by multiplying the target thickness $W$ in units of $\mu \mathrm{g} \mathrm{cm}^{-2}$. All of the $F W$ and $B W$ values obtained in the present work were found to be independent of $E_{0}$ above $600 \mathrm{MeV}$. In our previous papers, ${ }^{5-7}$ yield measurements of the photospallation reactions of various targets ranging from ${ }^{\text {nat }} \mathrm{V}$ to ${ }^{197} \mathrm{Au}$ were reported. From the slope parameter $P$ of the mass-yield curve in the empirical charge and mass distribution formula (CDMD), ${ }^{39}$ which is an indirect measure of the average excitation energy transferred in the spalling nuclei, it was found that the excitation energy seems to saturate at energies higher than $600 \mathrm{MeV}$. The $E_{0}$-independece of $F W$ and $B W$ obtained in this recoil study is consistent with that of the slope parameter $P$, and indicates that the photons responsible for the production of these nuclides are mostly of energies lower than $600 \mathrm{MeV}$. Therefore, the following discussion proceeds based on the average quantities above $600 \mathrm{MeV}$.

As an example, the averaged values of $F W$ and $B W$ are shown by open and closed circles, respectively, as a function of mass difference $\Delta A$ between a product $\left(A_{\mathrm{p}}\right)$ and a target $\left(A_{\mathrm{t}}\right)$ in Figure 1a for ${ }^{\text {nat }} \mathrm{Cu}$ and Figure $1 \mathrm{~b}$ for ${ }^{197} \mathrm{Au}$. The $F W$ values are higher than the corresponding $B W$, and both increase in parallel with an increase of $\Delta A$. This increasing trend with $\Delta A$ can be divided into two components: one is a steep increase for 

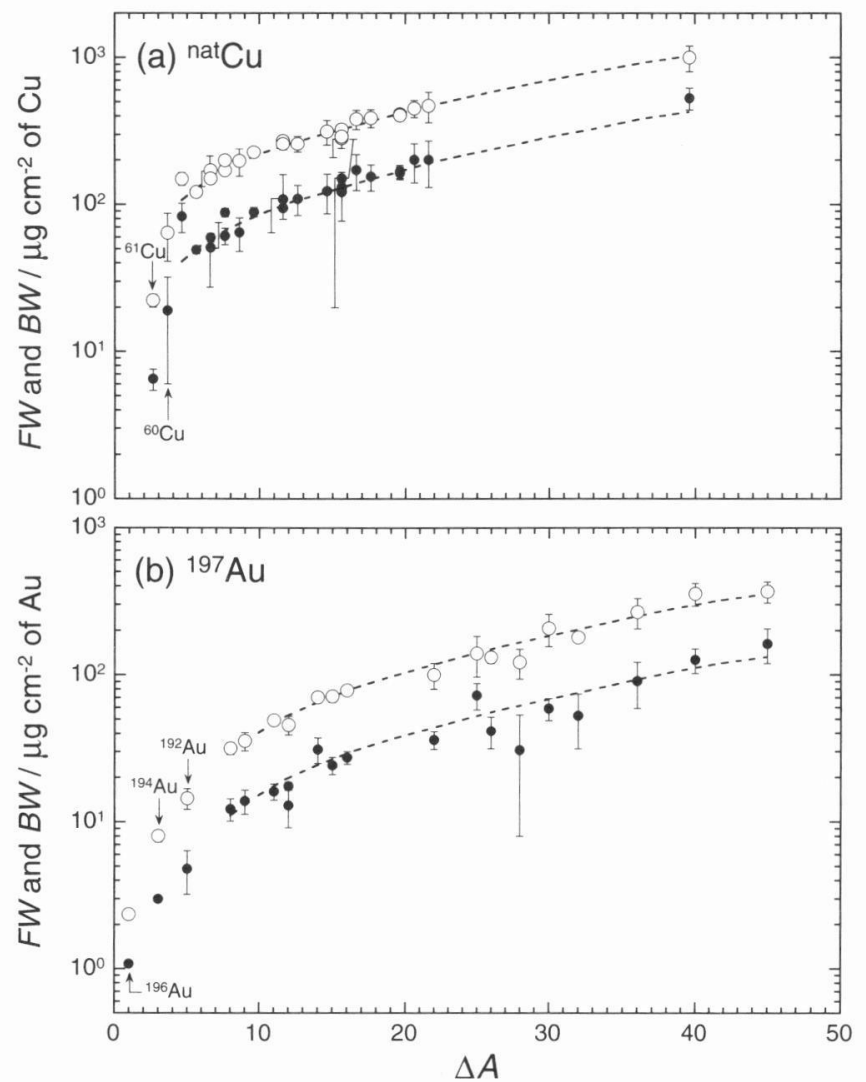

Figure 1. Averaged mean ranges $F W$ (open symbols) and $B W$ (closed symbols) at $E_{0} \geq 600 \mathrm{MeV}$ as a function of mass difference $\Delta A$ for (a) ${ }^{\text {nat }} \mathrm{Cu}$ and (b) ${ }^{197} \mathrm{Au}$. The dashed curves are to guide the eye. (From Reference 21)

the $(\gamma, x \mathrm{n})(x \geq 1)$ products mainly produced by GDR and the other is a gentle increase for the $(\gamma, x$ nyp $)(x, y \geq 1)$ products mainly produced by $\Delta$ resonance. However, this difference is not very clear for the $(\gamma, x \mathrm{n})$ reactions with higher multiplicities of $x \geq 4$, due to negligibly small contributions of GDR (see also Figure 1 in Reference 21 for ${ }^{\text {nat }} \mathrm{V},{ }^{93} \mathrm{Nb}$, ${ }^{\text {nat }} \mathrm{Ag}$, and ${ }^{\text {nat }} \mathrm{Ta}$ ). The existence of these two components in photospallation was previously suggested in yield measurements, ${ }^{6,7}$ in which the reaction yields of the $(\gamma, x \mathrm{n})(x \geq 1)$ products deviate from those deduced by the CDMD formula; ${ }^{39}$ a new empirical expression for this type of reaction was proposed.

The forward-to-backward ratios $F / B$ at $E_{0} \geq 600 \mathrm{MeV}$ are plotted as a function of $\Delta A$ in Figure 2 for ${ }^{\text {nat }} \mathrm{V},{ }^{\text {nat }} \mathrm{Cu},{ }^{93} \mathrm{Nb}$, ${ }^{\text {nat }} \mathrm{Ag}$, ${ }^{\text {nat }} \mathrm{Ta}$, and ${ }^{197} \mathrm{Au}$. As is expected from the parallel increases of $F W$ and $B W$ in Figure 1, the $F / B$ ratios are now shown to be independent of $\Delta A$, and their weighted mean values are $2.3 \pm 0.9$ for ${ }^{\text {nat }} \mathrm{V}, 2.4 \pm 0.5$ for ${ }^{\text {nat }} \mathrm{Cu}, 2.8 \pm 0.5$ for ${ }^{93} \mathrm{Nb}$, $2.7 \pm 0.4$ for ${ }^{\text {nat }} \mathrm{Ag}, 2.9 \pm 0.4$ for ${ }^{\text {nat }} \mathrm{Ta}$, and $2.5 \pm 0.6$ for ${ }^{197} \mathrm{Au}$. Noted is that these average values are all equal irrespective of the target within the associated errors. On the other hand, it was found from a literature survey that the $F / B$ ratios of typical spallation products from proton-induced reactions at incident proton energies of $E_{\mathrm{p}} \leq 3 \mathrm{GeV}$ vary with $\Delta A$, and decrease with an increase of $E_{\mathrm{p}}$, but become $E_{\mathrm{p}}$ - and $\Delta A$-independent above 3 $\mathrm{GeV}$. It seems interesting that this constant $F / B$ ratio of proton-induced reactions at $E_{\mathrm{p}}>3 \mathrm{GeV}$ is equal to that of the photon-induced reactions at $E_{0} \geq 600 \mathrm{MeV}$. The implications of this behavior are discussed in the following section in terms of the two-step vector velocity analysis.

4.1.2. Analysis by the Two-Step Vector Velocity Model. The measured recoil data may be used to derive some recoil parameters by means of the vector velocity model embodying the two-step mechanism commonly invoked in high-energy proton reactions. In the present work, the two-step vector velocity model developed by Winsberg ${ }^{40}$ was employed for this purpose. According to this model, the observable velocity $v_{1}$ of a
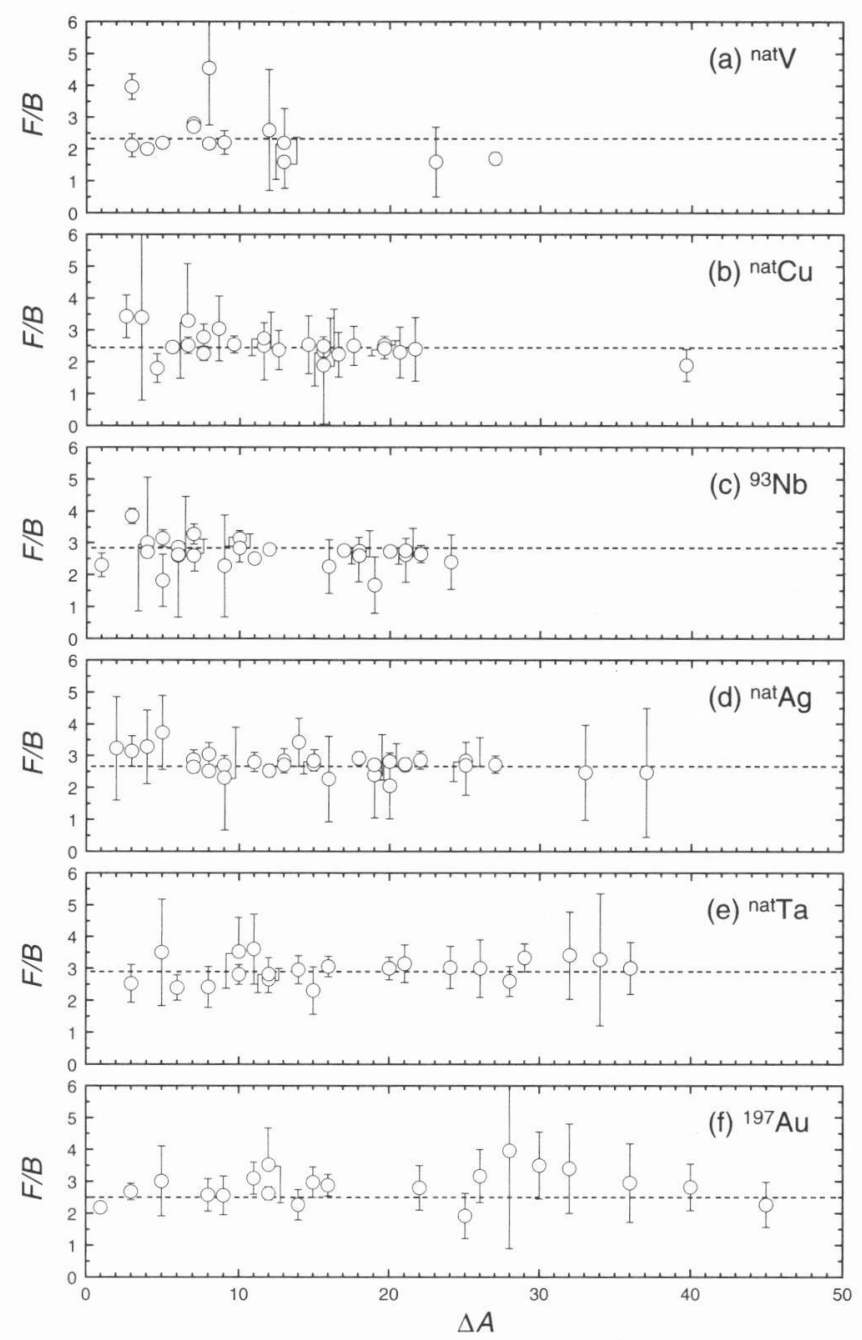

Figure 2. Forward-to-backward ratios $F / B$ at $E_{0} \geq 600 \mathrm{MeV}$ as a function of $\Delta A$ for (a) ${ }^{\text {nat }} \mathrm{V}$, (b) ${ }^{\text {nat }} \mathrm{Cu}$, (c) ${ }^{93} \mathrm{Nb}$, (d) ${ }^{\text {nat }} \mathrm{Ag}$, (e) ${ }^{\text {nat }} \mathrm{Ta}$, and (f) ${ }^{197} \mathrm{Au}$. (From Reference 21)

recoiling residual nucleus is resolved into two velocity components, $v$ and $V$, corresponding to the cascade step and the deexcitation or breakup step of the reaction, respectively. The cascade component $v$ is assumed to be parallel to the beam direction. The velocity $V$ must be symmetric in the moving system, and is assumed to be isotropic. Furthermore, if the range of a recoiling nucleus in the target material $R_{\mathrm{I}}$ can be related to its velocity by an expression of $R_{1}=C v_{1}^{N}$, where $C$ and $N$ are constants, and if $v / V$ is small, the following relations can be derived:

$$
\begin{aligned}
& F W=\frac{R_{0}}{4}\left\{1+\frac{2(N+2) v}{3} \frac{v}{V}+\frac{(N+1)^{2}}{4}\left(\frac{v}{V}\right)^{2}\right\}, \\
& B W=\frac{R_{0}}{4}\left\{1-\frac{2(N+2) v}{3} \frac{v}{V}+\frac{(N+1)^{2}}{4}\left(\frac{v}{V}\right)^{2}\right\} .
\end{aligned}
$$

The quantity $R_{0}$ is the mean range in the target material corresponding to the recoil velocity $V$, so that $R_{0}=C V^{N}$. The $R_{0}$ and $v / V$ were calculated from the measured quantities of $F W$ and $B W$ by using above two equations. The calculated $R_{0}$ was converted to the kinetic energy $T$ of the residual nucleus by using the range-energy table. The range-energy relation used to convert $R_{0}$ to $T$ and to obtain the values $C$ and $N$ was calculated by the SRIM2000 code developed by Ziegler et al. ${ }^{41}$ The forward velocity $v$ was calculated from the $v / V$ and $T$ values.

4.1.3. Mean Range $R_{0}$ and Kinematic Parameters $v$ and $T$. In Figure 3 the mean ranges $R_{0}$ in units of $\mathrm{mg} \mathrm{cm} \mathrm{cm}^{-2}$ of target foil of the $\left(\gamma, x\right.$ nyp) $(x, y \geq 1)$ products at $E_{0} \geq 600 \mathrm{MeV}$ are shown as a function of $\Delta A$ by different symbols for ${ }^{\text {nat }} \mathrm{V},{ }^{\text {nat }} \mathrm{Cu}$, ${ }^{93} \mathrm{Nb}$, ${ }^{\text {nat }} \mathrm{Ag},{ }^{\text {nat }} \mathrm{Ta}$, and ${ }^{197} \mathrm{Au}$. $R_{0}$ increases smoothly with an 


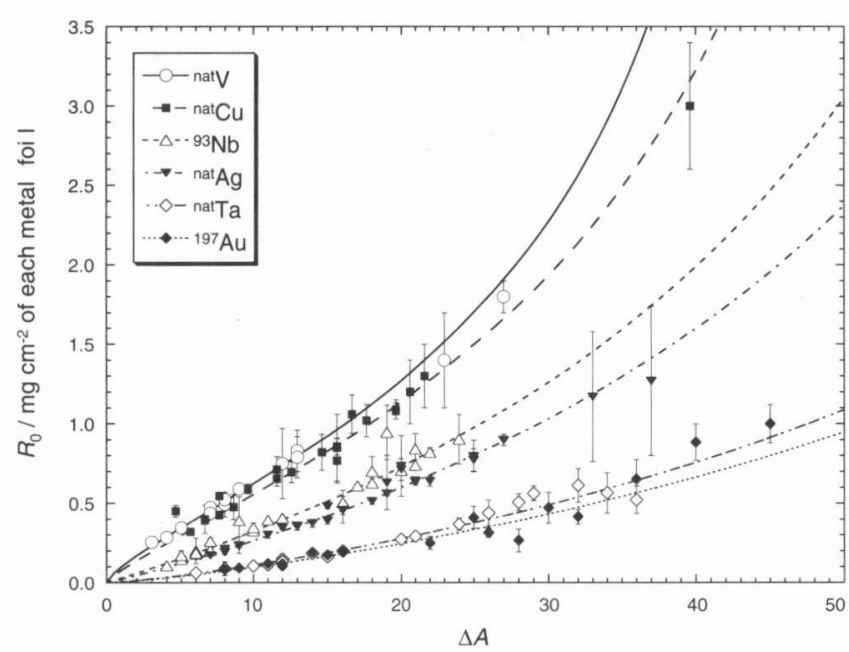

Figure 3. Mean ranges $R_{0}$ in the second step at $E_{0} \geq 600 \mathrm{MeV}$ as a function of $\Delta A$ for ${ }^{\text {nat }} \mathrm{V}$ (open circles), ${ }^{\text {nat }} \mathrm{Cu}$ (closed squares), ${ }^{93} \mathrm{Nb}$ (open triangles), ${ }^{\text {nat }} \mathrm{Ag}$ (closed inverse triangles), ${ }^{\text {nat }} \mathrm{Ta}$ (open diamonds), and ${ }^{197} \mathrm{Au}$ (closed diamonds). (From Reference 21)

increase of $\Delta A$ as shown by different lines for the respective targets in Figure 3. It was found that $R_{0}$ is well reproduced by a relation $R_{0}=a\left(A_{1} / \Delta A-1\right)^{-b}$ and that parameters $a$ and $b$ obtained by the least-squares fittings are a smooth function of atomic number of target $Z_{\mathrm{t}}{ }^{21}$ This relationship may be used to predict the unmeasured mean ranges and to estimate the recoil losses from target foils for the typical photospallations on various targets. Also, this systematic is available for protoninduced spallation, irrespectively of $E_{\mathrm{p}}$, due to the same $R_{0}$ (and $T$ ) values in proton-induced reaction as those in photon reactions.

The forward velocity $v$ is a good parameter to estimate the momentum transferred to an intermediate nucleus in the first cascade step and is related to the deposited excitation energy. On the other hand, the average recoil energy $T$ imparted to a residual recoiling nucleus is a convenient parameter to investigate the second evaporation step of the reaction. As an example, the variations of $v$ and $T$ at $E_{0} \geq 600 \mathrm{MeV}$ are shown as a function of $\Delta A$ by large open circles for ${ }^{\text {nat }} \mathrm{Cu}$ in Figures $4 \mathrm{a}$ and $4 \mathrm{~b}$, respectively. The cascade velocity $v$ increases linearly with an increase of $\Delta A$, indicating that the higher excitation energy is deposited on an intermediate nucleus to form a residual nucleus with a larger $\Delta A$. The kinetic energy $T$ also increases almost linearly with an increase of $\Delta A$, as explained by a random-walk theory. ${ }^{13}$ Proton results on ${ }^{\text {nat }} \mathrm{Cu}$ (Refs. 42 $47)$ are available at various proton energies $\left(E_{\mathrm{p}}\right)$, as indicated in the inset of Figure $4 \mathrm{~b}$. The $v$ values at $E_{\mathrm{p}} \leq 3 \mathrm{GeV}$ (open symbols) are apparently higher than those of the photon reactions and decrease with an increase of $E_{\mathrm{p}}$ up to $3 \mathrm{GeV}$. The $v$ values at $E_{\mathrm{p}}>3 \mathrm{GeV}$, indicated by closed symbols, are almost the same as those of the photon reactions. On the other hand, all of the $T$ values of the proton reactions are about the same as those of the photon reactions, irrespective of $E_{\mathrm{p}}$.

From a comparison with the proton results for other targets of ${ }^{27} \mathrm{Al}$, ${ }^{\text {nat }} \mathrm{V}$, ${ }^{\text {nat }} \mathrm{Ag}$, ${ }^{\text {nat }} \mathrm{Ta}$, and ${ }^{197} \mathrm{Au},{ }^{20,21}$ it is concluded that for typical spallation the $v$ values of proton reactions at $E_{\mathrm{p}} \leq 3$ $\mathrm{GeV}$ are higher than those of photon reactions at $E_{0} \geq 600$ $\mathrm{MeV}$ and decrease with an increase of $E_{\mathrm{p}}$ up to around $E_{\mathrm{p}}=3$ $\mathrm{GeV}$, and become constant at higher energies. This distinct difference of the $v$ values in the two types of nuclear reaction at the lower-energy region may be attributed to the lower momentum transferred in the initial electromagnetic interaction in photon reactions. The steep decrease of $v$ in proton reactions at $E_{\mathrm{p}} \leq 3 \mathrm{GeV}$ is due to the increasing nuclear transparency to the incoming proton; the difference disappears at proton energies above $3 \mathrm{GeV}$, where the momentum transfer in proton reactions is almost the same as that in photon reactions.
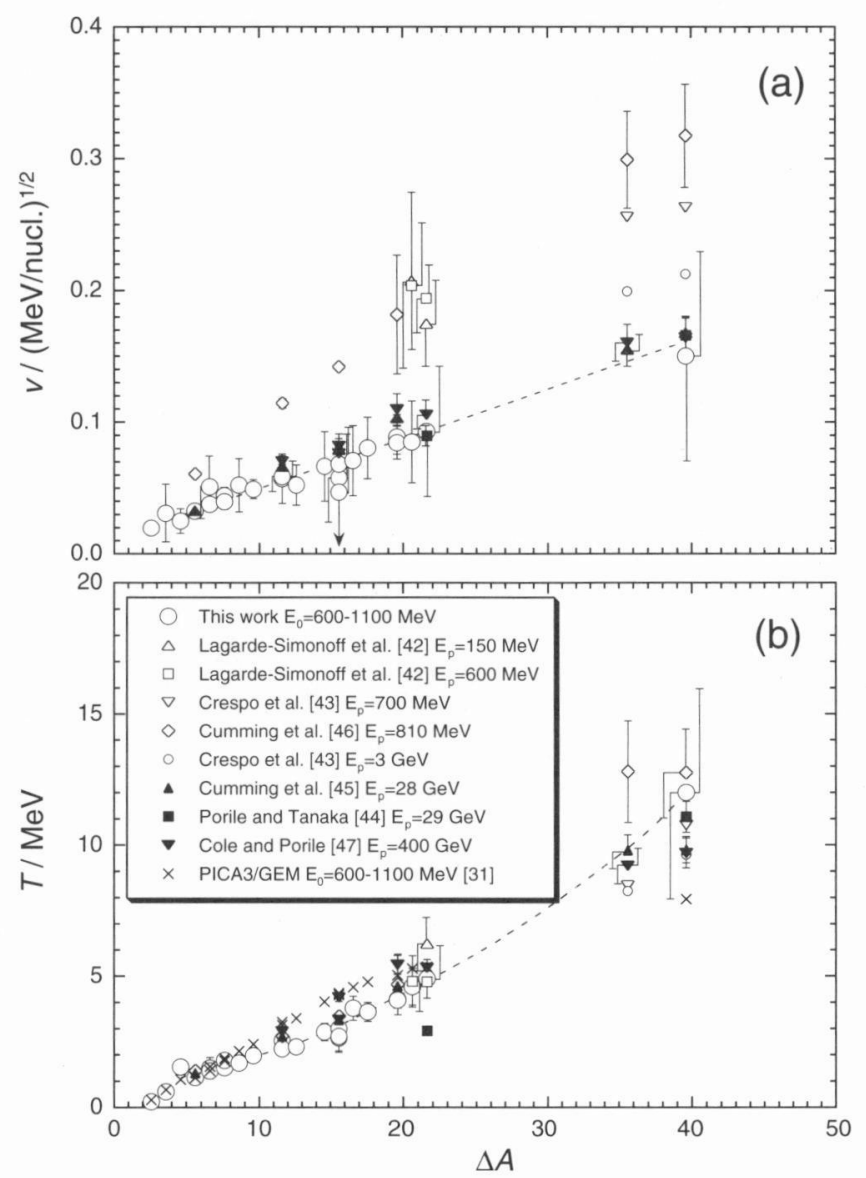

Figure 4. (a) Forward velocity $v$ in the first step and (b) kinetic energy $T$ in the second step as a function of $\Delta A$ for ${ }^{\text {nat }} \mathrm{Cu}$. See text for the details. (From Reference 33)

On the other hand, $T$ in proton reactions seems to be independent of $E_{\mathrm{p}}$ in the energy region cited above and agrees well with those of photon reactions at $E_{0} \geq 600 \mathrm{MeV}$. This consistency of the $T$ values suggests that the mechanism of the second deexcitation step is very similar in both photon and proton reactions.

The reproducibility of the kinetic energies $T$ by the PICA3/GEM code ${ }^{31}$ was examined. The calculated $T$ values for ${ }^{\text {nat }} \mathrm{Cu}$ at $E_{0}=600-1100 \mathrm{MeV}$ are indicated by crosses for the nuclides corresponding to the measured ones in Figure 4b. The calculated $T$ values increase with an increase of $\Delta A$ in a similar way as the experimental results. In order to check the reproducibility of the PICA3/GEM code ${ }^{31}$ quantitatively, the ratios of the calculated $T$ to the experimental $T$, $T$ (Cal.)/T(Exp.), are shown as a function of $\Delta A$ for ${ }^{\text {nat }} \mathrm{V},{ }^{\text {nat }} \mathrm{Cu}$, ${ }^{93} \mathrm{Nb}$, ${ }^{\text {nat }} \mathrm{Ag}$, ${ }^{\text {nat }} \mathrm{Ta}$, and ${ }^{197} \mathrm{Au}$ in Figure 5. The dashed lines represent $T(\mathrm{Cal}.) / T($ Exp. $)=1$. The PICA3/GEM code ${ }^{31}$ reproduces the experimental results for ${ }^{\text {nat }} \mathrm{V}$, ${ }^{\text {nat }} \mathrm{Cu},{ }^{93} \mathrm{Nb}$, and ${ }^{\text {nat }} \mathrm{Ag}$ fairly well, while the code underestimates the experiments for the heavier targets of nat $\mathrm{Ta}$ and ${ }^{197} \mathrm{Au}$. The underestimation of $T$ by the PICA3/GEM code ${ }^{31}$ in the heavy target region implys that the smaller separation energies of emitted nucleons are assumed for heavy targets in the calculation. The discrepancy may be explained by nuclear medium effects that would become progressively significant in heavier nuclei ${ }^{4}$ the smooth variation of the $\left(\gamma, \pi^{-} x \mathrm{n}\right)$ yields as a function of the neutron-to-proton ratio of the target $(N / Z)_{t}$ changes its characteristics at $(N / Z)_{\mathrm{t}}=1.30-1.40$, corresponding to $A_{\mathrm{t}}=100-130$, implying higher excitation energies due to progressively larger medium effects in nuclei with $A_{\mathrm{t}}>100$.

4.1.4. Systematization of the Second Step. Winsberg ${ }^{13}$ suggested that $\varepsilon_{\mathrm{s}}=T /\left(\triangle A / A_{\mathrm{t}}\right)$, which represents the average energy carried off by an evaporated nucleon, is a good parameter to systematize the second step, and found that $\varepsilon_{\mathrm{s}}$ is independent of 


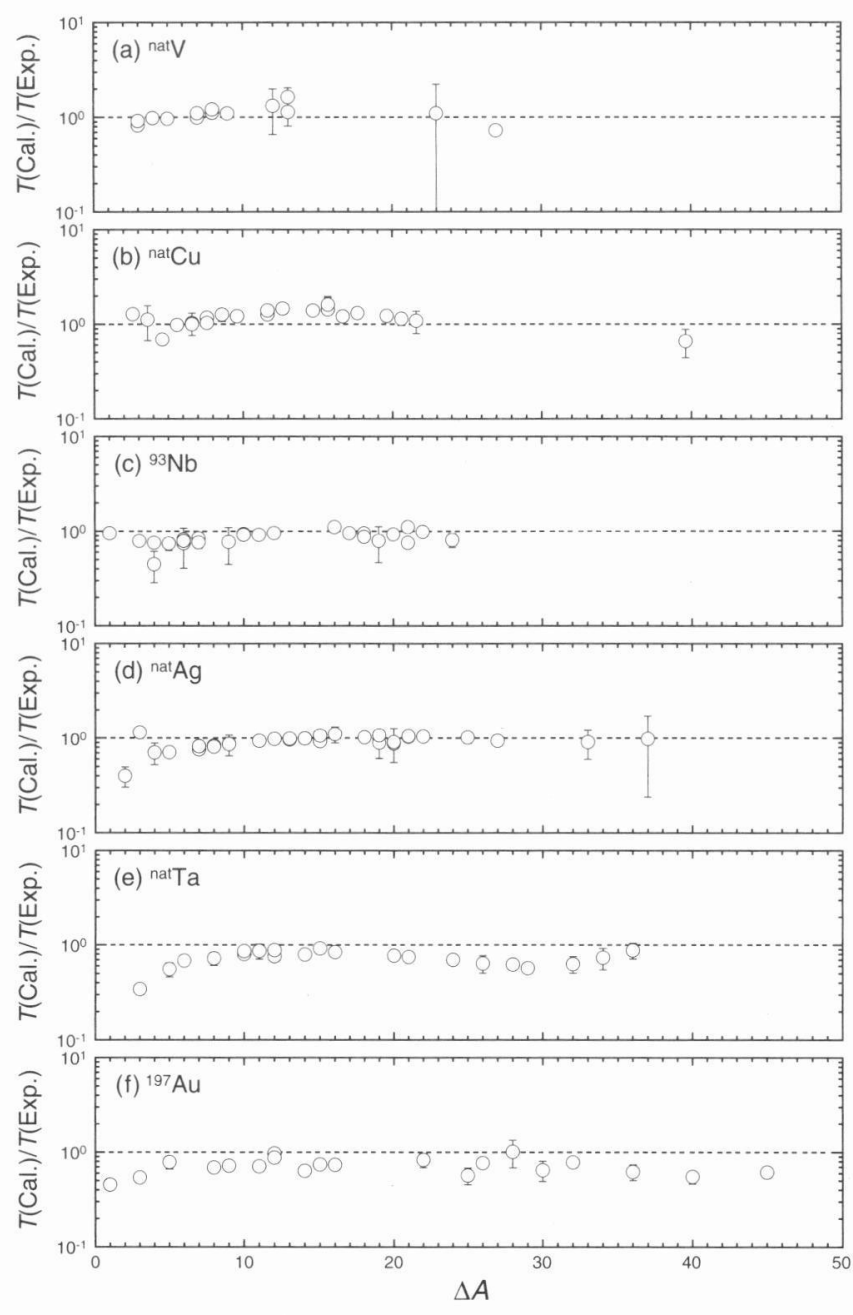

Figure 5. Ratio of the calculated $T$ by the PICA3/GEM code ${ }^{31}$ to the experimental $T$ at $E_{0} \geq 600 \mathrm{MeV}, T$ (Cal.) $/ T$ (Exp.), as a function of $\Delta A$ for (a) ${ }^{\text {nat }} \mathrm{V}$, (b) ${ }^{\text {nat }} \mathrm{Cu}$, (c) ${ }^{93} \mathrm{Nb}$, (d) ${ }^{\text {nat }} \mathrm{Ag}$, (e) ${ }^{\text {nat }} \mathrm{Ta}$, and (f) ${ }^{197} \mathrm{Au}$. (From Reference 33)

$\triangle A / A_{\mathrm{t}}$ and also of $A_{\mathrm{t}}$ for typical spallation reactions induced by protons. We applied this systematization for the photospallation. The $\varepsilon_{\mathrm{s}}$ values of the $(\gamma, x \mathrm{n})$ products increase with an increase of $\triangle A / A_{\mathrm{t}}$ and they are obviously lower than those of the $\left(\gamma, x\right.$ nyp) products. The latter is independent of $\Delta A / A_{t}$, as in proton reactions, and the weighted mean values are $13 \pm 2$, $13 \pm 3,17 \pm 3,16 \pm 1,15 \pm 4$, and $16 \pm 5 \mathrm{MeV}$ for ${ }^{\text {nat }} \mathrm{V},{ }^{\text {nat }} \mathrm{Cu},{ }^{93} \mathrm{Nb}$, ${ }^{\text {nat }} \mathrm{Ag}$, ${ }^{\text {nat }} \mathrm{Ta}$, and ${ }^{197} \mathrm{Au}$, respectively. The average $\varepsilon_{\mathrm{s}}$ values are plotted in Figure 6 as a function of $A_{t}$ by open circles together with those of the proton reaction $\mathrm{s}^{4-57}$ by closed squares. The details for the proton data were described in Reference 21 . The $\varepsilon_{\mathrm{s}}$ values of both the photon and proton reactions increase slightly with an increase of $A_{\mathrm{t}}$ up to around $A_{\mathrm{t}}=100$ and become almost constant at heavier targets. This $A_{\mathrm{t}}$-dependent feature of $\varepsilon_{\mathrm{s}}$ has not been reported previously as far as we are aware.

It was reported ${ }^{5,7}$ that there are linear relationships between the neutron-to-proton ratios of the most probable product, $(N / Z)_{\mathrm{p}}$, and those of targets, $(N / Z)_{\mathrm{t}}$, both in the photon- and proton- (and $\alpha-)$ spallation of ${ }^{\text {nat }} \mathrm{V}^{-}{ }^{197} \mathrm{Au}$. The slope of $(N / Z)_{\mathrm{p}}$ against $(N / Z)$, for the photospallation is steeper than that for proton- (and $\alpha-)$ spallation, and the $(N / Z)_{\mathrm{p}}$ of photospallation is shifted to the more neutron rich side for the targets of $(N / Z)_{t}>$ 1.2 with respect to that of proton- (and $\alpha-$ ) spallation. This suggests that the average excitation energy of cascade residues in photospallation is lower than that in hadron spallation. The $\varepsilon_{\mathrm{s}}$ values of the photon reactions obtained in the present work are almost equal to those of the proton reactions for ${ }^{27} \mathrm{Al}$ of $(N / Z)_{\mathrm{t}}=1.08$ and ${ }^{\text {nat }} \mathrm{Cu}$ of $(N / Z)_{\mathrm{t}}=1.19$, but systematically lower for the targets of $(N / Z)_{t}>1.2$, such as ${ }^{\text {nat }} \mathrm{Ag}$ of $(N / Z)_{\mathrm{t}}=$

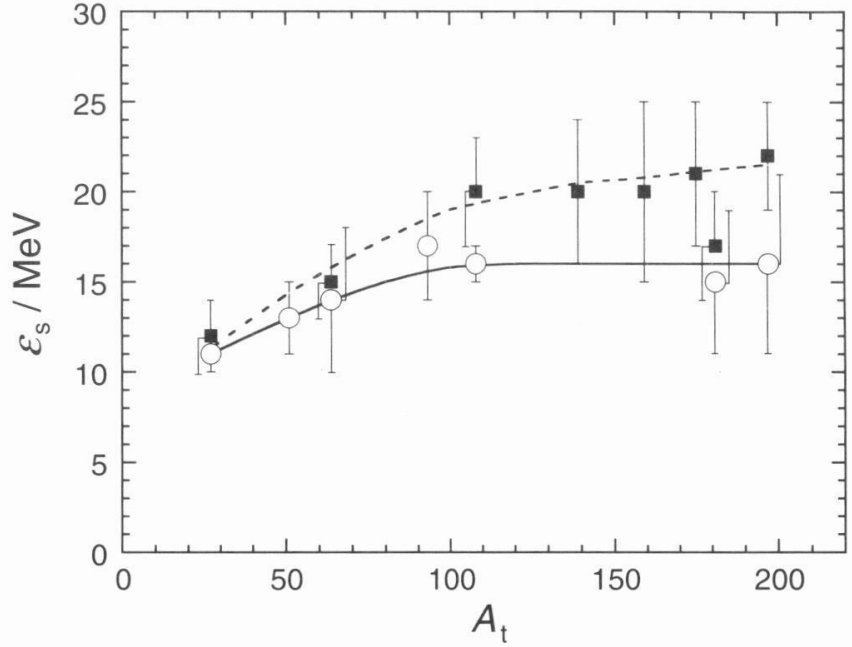

Figure 6. Parameter $\varepsilon_{\mathrm{s}}$ as a function of $A_{1}$ for photon reaction (open circles connected by a solid line) and proton reaction (closed squares connected by a dashed line). (From Reference 21)

$1.30,{ }^{\text {nat }} \mathrm{Ta}$ of $(N / Z)_{\mathrm{t}}=1.48$, and ${ }^{197} \mathrm{Au}$ of $(N / Z)_{\mathrm{t}}=1.49$. This variation of $\varepsilon_{\mathrm{s}}$ shown in Figure 6 is consistent with the abovementioned findings of $A_{1}$-dependent features of the photospallation and photopion reaction yields, ${ }^{4,7}$ though fairly large errors are accompanied with the $\varepsilon_{\mathrm{s}}$ values.

\subsection{Photofission of ${ }^{197} \mathrm{Au}$ and ${ }^{209} \mathrm{Bi}$}

4.2.1. Recoil Properties of the Photofission Products from ${ }^{197} \mathrm{Au}$. The 29 radionuclides with $A=24-131$ produced from ${ }^{197} \mathrm{Au}$ were identified in the target and catcher foils in addition to the 20 with $A=152-197$ cited above. Their effective mean ranges $F W$ and $B W$ were also found to be independent of $E_{0}$ at the studied $E_{0}$. The $F / B$ ratios at $E_{0} \geq 600 \mathrm{MeV}$ are plotted by open circles as a function of mass difference $\Delta A$ in Figure $7 \mathrm{a}$. The $F / B$ ratio represents the extent of forward peaking of the recoil, and thus is a simplified measure of the angular distribution of the product nuclei. As mentioned in sect. 4.1.1, the $F / B$ ratio for typical photospallation products is independent of $\Delta A$ and also of $A_{\mathrm{t}}$, and the weighted mean value for ${ }^{197} \mathrm{Au}$ at $\Delta A \leq$ 45 is $2.5 \pm 0.6$ as indicated by a dashed line in Figure $7 \mathrm{a}$. The $F / B$ ratios at $\Delta A \geq 66$ are also independent of $\Delta A$, but the weighted mean value of $F / B=1.1 \pm 0.1$ shown by a solid line in Figure $7 \mathrm{a}$ is apparently lower than that for the photospallation products at $\Delta A \leq 45$, indicating the isotropic angular distribution of the product nuclei. The proton results ${ }^{47,53,55,58}$ are also included in Figure 7a by the different symbols as shown in the inset of the figure. The $F / B$ ratios at $E_{\mathrm{p}}=0.58,1$, and $3 \mathrm{GeV}$, which are shown by open symbols, are apparently higher than those at the higher $E_{\mathrm{p}}$ (closed symbols) and also those of the photon reaction (open circles). The $F / B$ ratios at $E_{\mathrm{p}} \leq 3 \mathrm{GeV}$ increase with an increase of $\Delta A$ and reach peaks around $\Delta A=$ 40 at $E_{\mathrm{p}}=1 \mathrm{GeV}$ and around $\Delta A=55$ at $E_{\mathrm{p}}=3 \mathrm{GeV}$. After the peak, the $F / B$ decreases with an increase of $\Delta A$ and almost levels off at $\Delta A \geq 87$. The $F / B$ values of the proton reactions decrease with an increase of $E_{\mathrm{p}}$ up to $E_{\mathrm{p}}=3 \mathrm{GeV}$, but becomes $E_{\mathrm{p}}$-independent at the higher $E_{\mathrm{p}}$ (closed symbols). It is noted that the $E_{\mathrm{p}}$-independent $F / B$ values of the proton reactions at $E_{\mathrm{p}}$ $>3 \mathrm{GeV}$ are equal to those of the photon reaction at $E_{0}=$ $600-1100 \mathrm{MeV}$ except for the results of ${ }^{196} \mathrm{Au}$ and ${ }^{194} \mathrm{Au}$ produced by the direct reactions.

The kinetic energies $T$ of the product nuclei were evaluated based on the same procedure as described in sect. 4.1.2. In Figure $7 \mathrm{~b}$, the $T$ values at $E_{0} \geq 600 \mathrm{MeV}$ are shown as a function of $\Delta A$ by open circles. At $\Delta A \leq 45$ the $T$ increases with an increase of $\Delta A$, showing the two components; one is a steep increase for the $(\gamma, x \mathrm{n})(x \geq 1)$ products ${ }^{196,194,192} \mathrm{Au}$ (a dotted curve), and the other is a gradual increase for the $(\gamma, x$ nyp) $(x, y$ $\geq 1$ ) products (a solid curve). The increasing trend for the 

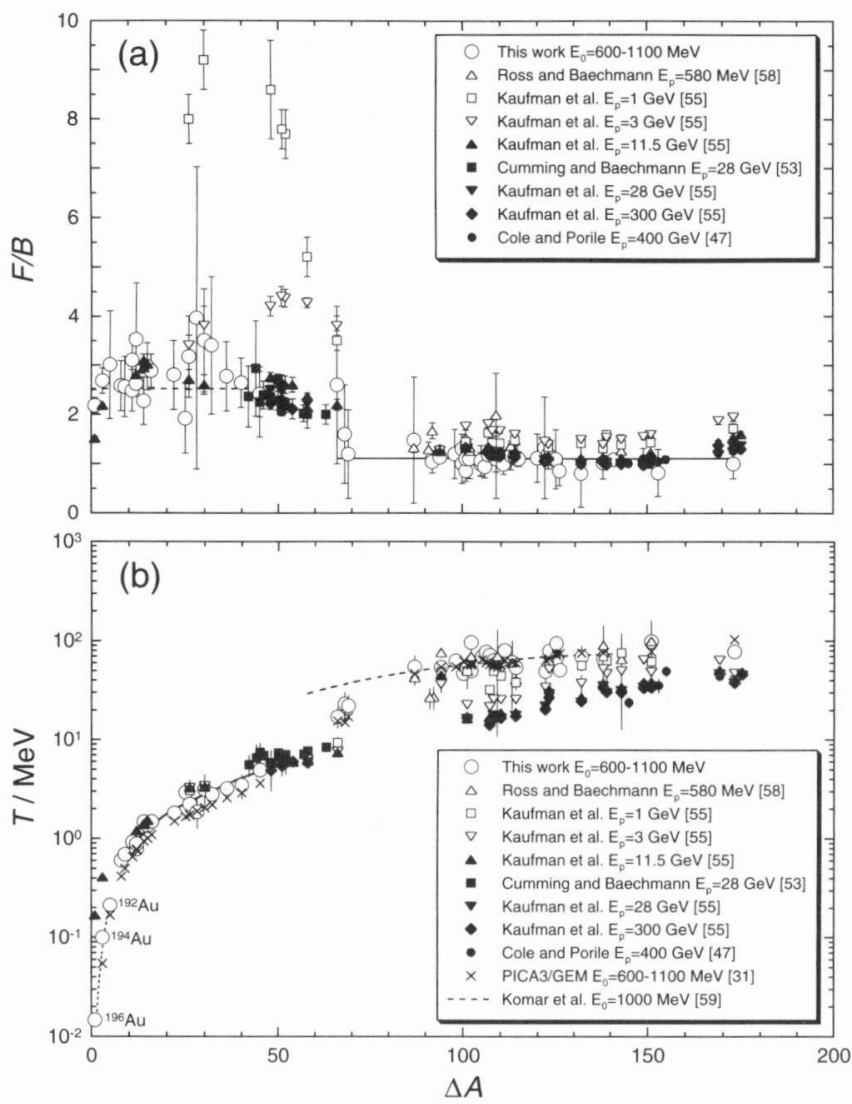

Figure 7. (a) Forward-to-backward ratios $F / B$ and (b) kinetic energy $T$ for ${ }^{197} \mathrm{Au}$ at $E_{0} \geq 600 \mathrm{MeV}$ as a function of $\Delta A$. See text for the details. (From References 25 and 34)

latter component is well explained in terms of a random-walk theory for typical spallation ${ }^{13}$ (see sect. 4.1.4). In this theory, the $\varepsilon_{\mathrm{s}}$ values are independent of $\Delta A / A_{\mathrm{t}}$ and the average $\varepsilon_{\mathrm{s}}$ value is $16 \pm 5 \mathrm{MeV}$. On the other hand, the $T$ values at $\Delta A>45$ are quite higher than those extrapolated from the spallation trend at $\Delta A \leq 45$. The $\varepsilon_{\mathrm{s}}$ values are more than $50 \mathrm{MeV}$, implying the different production mechanism from typical spallation. Komar et al. ${ }^{59}$ measured the kinetic energies of coincident fragment pairs in the photofission of ${ }^{197} \mathrm{Au}$ at $E_{0}=1000 \mathrm{MeV}$ by using the semiconductor detectors. Their results are indicated by a dashed curve in Figure $7 \mathrm{~b}$. The $T$ values obtained radiochemically in the present work seem to be well consistent with the physical measurements by Komar et al. ${ }^{59}$ except for those of barium isotopes at $\Delta A=66,68$, and 69. The smaller $T$ values of $\mathrm{Ba}$ isotopes may indicate that their productions are based on both fission and spallation. The proton results ${ }^{47,53,55,58}$ are shown in Figure $7 \mathrm{~b}$ for a comparison. At $\Delta A \leq 66$ the $T$ values for the proton reactions are consistent with each other, irrespectively of $E_{\mathrm{p}}$, and also with those of the photon reaction at $E_{0} \geq 600 \mathrm{MeV}$ (see sect. 4.1.3). On the other hand, the $T$ values at $\Delta A>66$ decrease with an increase of $E_{\mathrm{p}}$ and become constant at $E_{\mathrm{p}}>3 \mathrm{GeV}$ (closed symbols). This decreasing trend is explained as a change in the reaction mechanism from fission to deep spallation. In case of the photon reaction, the effective photon energies are lower than $600 \mathrm{MeV}$ and the variation of $T$ is not found above $600 \mathrm{MeV}$. The $T$ values calculated by the PICA3/GEM code ${ }^{31}$ at $E_{0}=600-1100 \mathrm{MeV}$ are indicated by crosses in Figure $7 b$. The PICA3/GEM code ch $^{31}$ reproduces the $T$ values of the products with $\Delta A>45$, which are formed in the two-body breakup process, though the code underestimates by a factor of about 0.7 for the spallation products with $\Delta A \leq 45$ (see sect. 4.1.3).

4.2.2. Production of ${ }^{24} \mathrm{Na}$. Recoils of ${ }^{24} \mathrm{Na}$ make it possible to trace how the production mechanism of light nuclei changes with target mass $A_{\mathrm{t}}$. The $F / B$ ratios of ${ }^{24} \mathrm{Na}$ produced from ${ }^{27} \mathrm{Al},{ }^{\text {nat }} \mathrm{V}$, ${ }^{\text {nat }} \mathrm{Cu}$, ${ }^{\text {nat }} \mathrm{Ag}$, and ${ }^{197} \mathrm{Au}$ are shown in Figure $8 \mathrm{a}$ by
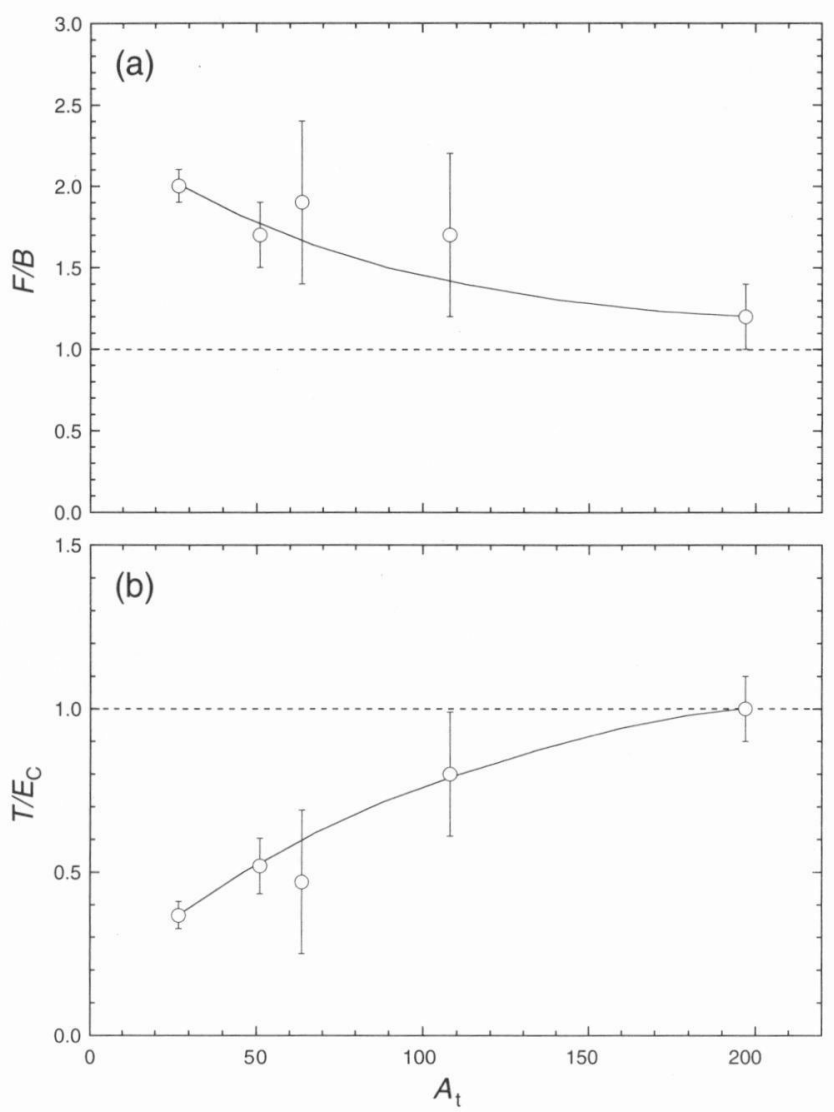

Figure 8. (a) Forward-to-backward ratios $F / B$ and (b) ratios $T / E_{\mathrm{C}}$ of ${ }^{24} \mathrm{Na}$ produced from ${ }^{27} \mathrm{Al},{ }^{\text {nat }} \mathrm{V},{ }^{\text {nat }} \mathrm{Cu},{ }^{\text {nat }} \mathrm{Ag}$, and ${ }^{197} \mathrm{Au}$ at $E_{0} \geq 600 \mathrm{MeV}$ as a function of the target mass number $A_{\mathrm{t}}$. (From Reference 25)

open circles as a function of $A_{\mathrm{t}}$. The $F / B$ values for ${ }^{27} \mathrm{Al}$, ${ }^{\text {nat }} \mathrm{V}$, and ${ }^{\text {nat }} \mathrm{Cu}$ are about 2 and they agree with the photospallation trend (see sect. 4.1.1). However, the $F / B$ values decrease gradually with an increase of $A_{t}$ as a whole and attain to almost unity for ${ }^{197} \mathrm{Au}$. This variation indicates that the change of the angular distribution of ${ }^{24} \mathrm{Na}$ from the forward-peaking distribution to the isotropic distribution when $A_{\mathrm{t}}$ increases.

It may also be interesting to compare the observed mean kinetic energy $T$ of ${ }^{24} \mathrm{Na}$ with the energy $E_{\mathrm{C}}$ acquired by Coulomb repulsion in the two-body breakup process. The ratio of $T$ to $E_{\mathrm{C}}$ would be small if ${ }^{24} \mathrm{Na}$ is produced as a residue of nucleon evaporation, and would be of the order of unity if the kinetic energy of ${ }^{24} \mathrm{Na}$ is acquired as a results of Coulomb repulsion in the two-body breakup process such as fission or fragmentation. Thus, the $T / E_{\mathrm{C}}$ ratios are shown as a function of $A_{\mathrm{t}}$ in Figure 8b. The $T / E_{\mathrm{C}}$ ratios are about 0.5 for ${ }^{27} \mathrm{Al},{ }^{\text {nat }} \mathrm{V}$, and ${ }^{\text {nat }} \mathrm{Cu}$, and are almost unity for ${ }^{\text {nat }} \mathrm{Ag}$ and ${ }^{197} \mathrm{Au}$.

The variations of $F / B$ and $T / E_{\mathrm{C}}$ with $A_{\mathrm{t}}$ may be explained as a result of the transition of the reaction mechanism from spallation to fission or fragmentation. As mentioned in sect. 4.1.4, the kinetic energy $T$ of typical photospallation products is well parameterized by $\varepsilon_{\mathrm{s}}$; the $\varepsilon_{\mathrm{s}}$ values are all in the range of 10-20 $\mathrm{MeV}$. The $\varepsilon_{\mathrm{s}}$ values for ${ }^{24} \mathrm{Na}$ from ${ }^{27} \mathrm{Al}$, ${ }^{\text {nat }} \mathrm{V}$, and ${ }^{\text {nat }} \mathrm{Cu}$ are $11.9 \pm 1.4,18.9 \pm 3.1$, and $21 \pm 10 \mathrm{MeV}$, respectively, and they agree with the spallation trend. However, the $\varepsilon_{\mathrm{s}}$ values of $51 \pm 13 \mathrm{MeV}$ for ${ }^{\text {nat }} \mathrm{Ag}$ and of $96 \pm 11 \mathrm{MeV}$ for ${ }^{197} \mathrm{Au}$ are quite high compared with those for the lighter targets, implying the different reaction mechanism from spallation. It is also noted that the existence of two nuclear processes in the photoproduction of ${ }^{24} \mathrm{Na}$ was found in the mass dependence of the reaction yields, $;^{9,10}$ the yields decrease exponentially with an increase of $A_{\mathrm{t}}$ (spallation), but suddenly change the slope to a gradual increase around $A_{\mathrm{t}}=100$ (fragmentation). Recently, the yields of ${ }^{7,10} \mathrm{Be},{ }^{22,24} \mathrm{Na}$, and ${ }^{28} \mathrm{Mg}$ from ${ }^{197} \mathrm{Au}$ were measured by the author's group. ${ }^{10}$ They are more than one order of magnitude higher than those extrapolated from the mass yield curve of 
fission, indicating a different reaction mechanism from fission; fragmentation.

4.2.3. Charge and Mass Yield Distributions. We measured the yields of 58 radionuclides with $A=42-131$ formed in the photofission of ${ }^{197} \mathrm{Au}$ at $E_{0}=300-1100 \mathrm{MeV}$. In the ${ }^{209} \mathrm{Bi}$ experiments, those of 63 nuclides with $A=56-135$ were obtained. All of the yields increase steeply with an increase of $E_{0}$ up to $600 \mathrm{MeV}$ and increase gradually above $600 \mathrm{MeV}$, indicating the resonance-type excitation function attributed to $\Delta$ resonance.

The charge distribution $Y_{\mathrm{CD}}(Z)$ among the isobars of a certain product mass number $A$ was assumed to be represented by the following Gaussian function:

$$
Y_{C D}(Z)=Y_{C D}\left(Z_{p}\right) \cdot \exp \left\{-\frac{\left(Z-Z_{p}\right)^{2}}{C_{Z}}\right\}
$$

where $Y_{\mathrm{CD}}\left(Z_{\mathrm{p}}\right)$ is the maximum yield of the charge distribution, $Z_{\mathrm{p}}$ being the most probable charge. The width parameter $C_{\mathrm{Z}}$ was assumed to be constant, irrespectively of $A$, and $Z_{\mathrm{p}}$ to be a linear function of $A$, i.e., $Z_{\mathrm{p}}=R A+S$. By assuming the unchanged charge distribution (UCD) hypothesis, the parameter $R$ is related to a charge-to-mass ratio of a fissioning nucleus as $R=Z_{\mathrm{t}} /\left(A_{\mathrm{t}}-V_{\mathrm{pre}}\right), v_{\mathrm{pre}}$ being the number of prefission neutrons. The parameter $S$ is a measure of the number of average post fission neutrons $v_{\text {post }}$ as $S=Z_{\mathrm{t}} v_{\text {post }} /\left(A_{\mathrm{t}}-v_{\text {pre }}\right)$ : Equation 2 was fitted to the experimental yields by a least squares method, and four free parameters $Y_{\mathrm{CD}}\left(Z_{\mathrm{p}}\right), C_{\mathrm{Z}}, R$, and $S$ were determined.
As an example, the charge distributions obtained for ${ }^{209} \mathrm{Bi}$ at $E_{0}=1000 \mathrm{MeV}$ are shown in Figure 9 by solid curves for the representative 10 mass chains of $A=72,83,86,88,95,96,97$, 103,105 , and 126 , in which more than two yield data are available. The independent yields (I), the cumulative yields by $\beta^{-}$ decay $(\mathrm{C}-)$, and by $\beta^{+}$decay and/or electron capture $(\mathrm{C}+)$ are shown by open circles, closed triangles, and closed squares, respectively. The cumulative yields are shown by a dotted curve for $\mathrm{C}-$ and by a dashed curve for $\mathrm{C}+$ by integrating the independent $Y_{\mathrm{CD}}(Z)$ curve. The results of these fittings are fairly good and the present assumptions in eq 2 are reasonable. The yields calculated by the PICA3/GEM code ${ }^{31}$ are compared by crosses in Figure 9. They show the symmetric charge distribution with almost the same width as the experiments. The maximum yield values are approximately comparable to the experimental ones, though slight differences in the peak position $\left(Z_{\mathrm{p}}\right)$ are shown; $Z_{\mathrm{p}}$ is reproduced well for $A=96-105$, but is overestimated for lower $A$ and underestimated for $A=$ 126. The more detailed comparison is given below referring to the charge distribution parameters.

The full width at half maximum of the charge distribution $F W H M_{\mathrm{CD}}$ in charge unit (c.u.) and the parameters $R$ and $S$ were found to be independent of $E_{0}$ above $600 \mathrm{MeV}$, and their weighted mean values are tabulated in Table 1 . The $F W H M_{\mathrm{CD}}$ values of ${ }^{197} \mathrm{Au}$ and ${ }^{209} \mathrm{Bi}$ are consistent with each other. The $R$ value of $0.424 \pm 0.001$ for ${ }^{197} \mathrm{Au}$ implies 11-neutron emission before fission $\left(v_{\mathrm{pre}}=11 \pm 1\right)$ as deduced from the UCD assumption. On the other hand, the $S$ value of $0.7 \pm 0.1$ implies the

TABLE 1: Charge and Mass Yield Distribution Parameters in the Photofission of ${ }^{197} \mathrm{Au}$ and ${ }^{209} \mathrm{Bi}$ at $E_{0} \geq 600 \mathrm{MeV}(\mathrm{From}$ Reference 34)

\begin{tabular}{ccccccc}
\hline & Target & $F W H M_{\mathrm{CD}}[\mathrm{c} . \mathrm{u}]$. & $R$ & $S$ & $A_{\mathrm{p}}[\mathrm{m} . \mathrm{u}]$. & $F W H M_{\mathrm{MD}}[\mathrm{m.u}]$. \\
\hline \multirow{2}{*}{${ }^{197} \mathrm{Au}$} & Exp. & $2.2 \pm 0.1$ & $0.424 \pm 0.001$ & $0.7 \pm 0.1$ & $92 \pm 1$ & $39 \pm 1$ \\
& - PICA3/GEM & $2.2 \pm 0.1$ & $0.423 \pm 0.001$ & $1.5 \pm 0.1$ & $86 \pm 1$ & $37 \pm 2$ \\
\hline \multirow{2}{*}{${ }^{209} \mathrm{Bi}$} & Exp. & $2.1 \pm 0.1$ & $0.421 \pm 0.001$ & $0.6 \pm 0.1$ & $96 \pm 1$ & $33 \pm 1$ \\
& PICA3/GEM & $2.6 \pm 0.1$ & $0.403 \pm 0.001$ & $2.6 \pm 0.1$ & $94 \pm 1$ & $34 \pm 1$ \\
\hline
\end{tabular}
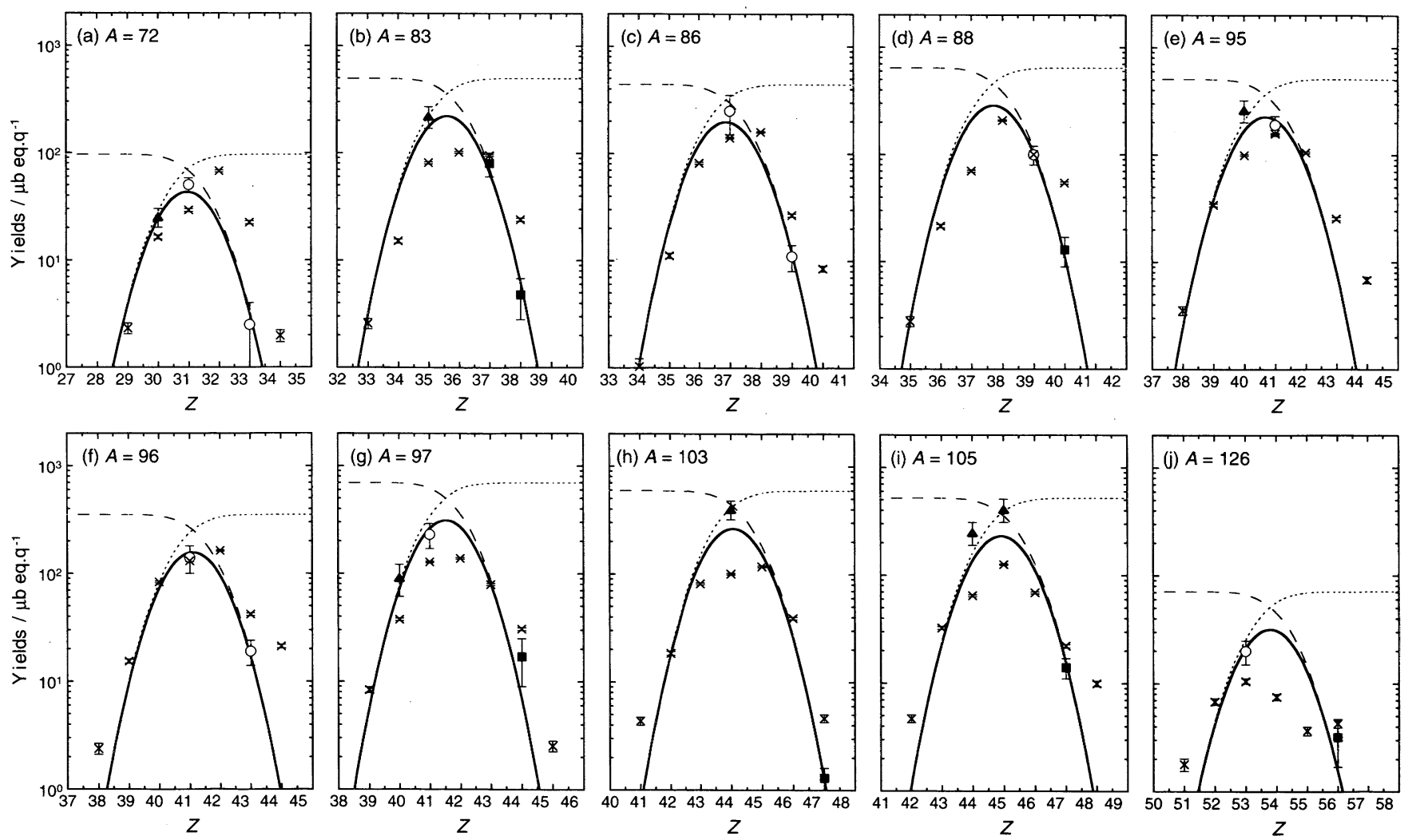

Figure 9. Charge distributions for mass chains of (a) 72, (b) 83, (c) 86, (d) 88, (e) 95, (f) 96, (g) 97, (h) 103 , (i) 105 , and (j) 126 at $E_{0}=1000 \mathrm{MeV}$. See text for the details. (From Reference 26) 
average $1.7 \pm 0.3$ post neutrons $\left(v_{\text {post }}\right)$. The $v_{\text {pre }}$ and $v_{\text {post }}$ values for ${ }^{209} \mathrm{Bi}$ are $12 \pm 1$ and $1.4 \pm 0.3$, respectively, and they are comparable with those for ${ }^{197} \mathrm{Au}$.

The $E_{0}$-independent trend of the charge distribution parameters at $E_{0} \geq 600 \mathrm{MeV}$ is reproduced by the PICA3/GEM code, ${ }^{31}$ and the average values of each parameter are compared in Table 1. The $F W H M_{\mathrm{CD}}$ value calculated for ${ }^{197} \mathrm{Au}$ is consistent with the experimental result, while that for ${ }^{209} \mathrm{Bi}$ is higher by 0.5 c.u. Also noted is that the $R$ value calculated for ${ }^{209} \mathrm{Bi}$ is quite smaller than the experimental one, indicating smaller $v_{\text {pre }}$ value in the calculation. On the other hand, the $S$ values for both ${ }^{197} \mathrm{Au}$ and ${ }^{209} \mathrm{Bi}$ are more than two times higher than the experiments. The overestimation of $S$ by the PICA3/GEM $\operatorname{code}^{31}$ means that a higher excitation energy of fission fragments is assumed in the calculation.

Based on the charge distribution parameters, the total chain yields in unit of $\mu \mathrm{b}$ per equivalent quanta ( $\mu \mathrm{b}$ eq.q. ${ }^{-1}$ ) were calculated. As an example, the total chain yields obtained at $E_{0}=1000 \mathrm{MeV}$ are shown in Figure 10 as a function of $A$ by open circles and open squares for ${ }^{197} \mathrm{Au}$ and ${ }^{209} \mathrm{Bi}$, respectively. These mass yield distributions are well reproduced by a Gaussian function as shown in Figure 10 by solid and dashed curves for ${ }^{197} \mathrm{Au}$ and ${ }^{209} \mathrm{Bi}$, respectively. The most probable mass $\left(A_{\mathrm{p}}\right)$ and the full width at half maximum $\left(F W H M_{\mathrm{MD}}\right)$ of the mass yield distribution were found to be independent of $E_{0}$ above $600 \mathrm{MeV}$, and the weighted mean values are tabulated in Table 1. The $A_{\mathrm{p}}$ value obtained for ${ }^{197} \mathrm{Au}$ is smaller by 4 m.u. than that for ${ }^{209} \mathrm{Bi}$, while the $F W H M_{\mathrm{MD}}$ is larger by 6 m.u. The total fission yield of $10 \pm 1 \mathrm{mb}$ eq.q. ${ }^{-1}$ for ${ }^{209} \mathrm{Bi}$ at $E_{0}=1000$ $\mathrm{MeV}$ is about three times higher than the value of $3.2 \pm 0.1 \mathrm{mb}$ eq.q. ${ }^{-1}$ for ${ }^{197} \mathrm{Au}$. These differences are considered to be important clues to the excitation energy and nuclear structure of fissioning preactinide nuclei in the neutron-deficient region.

The mass yields calculated by the PICA3/GEM code ${ }^{31}$ at $E_{0}$ $=1000 \mathrm{MeV}$ are shown Figure 10 by closed symbols. They also show the symmetric mass yield distribution. As mentioned above, however, large differences in the charge distribution parameters were found between the experimental results and the PICA3/GEM calculation. ${ }^{31}$ There should be some discrepancies in the meaning even if the $A_{\mathrm{p}}$ and/or $F W H M_{\text {MD }}$ values are reproduced by the PICA3/GEM calculation. ${ }^{31}$ The PICA3/GEM code ${ }^{31}$ employs the Generalized Evaporation Model $^{32}$ and the Rutherford Appleton Laboratory (RAL) model $^{38}$ to describe the competition between particle evaporation and fission. The predicted fission yields at intermediate energies strongly depend not only on the fission models, but also on quality of the models for the cascade and

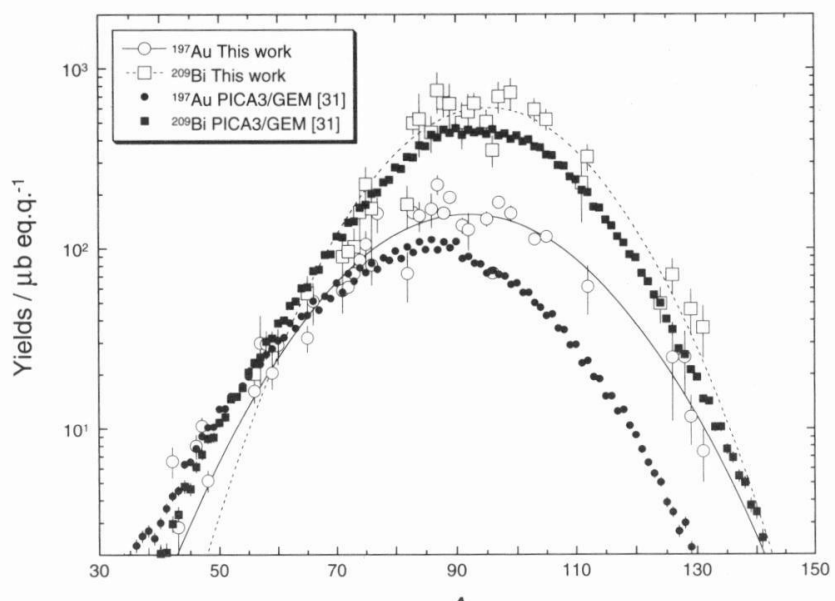

Figure 10. Mass yield curves of the photofission of ${ }^{197} \mathrm{Au}$ and ${ }^{209} \mathrm{Bi}$ at $E_{0}=1000 \mathrm{MeV}$. The date for ${ }^{197} \mathrm{Au}$ and ${ }^{209} \mathrm{Bi}$ are shown by open circles connected by a solid curve and by open squares connected by a dashed curve, respectively. The results of the PICA3/GEM calculations ${ }^{31}$ are plotted by closed circles and closed squares for ${ }^{197} \mathrm{Au}$ and ${ }^{209} \mathrm{Bi}$, respectively. (From References 26 and 34 ) preequilibrium steps that precede fission and the particle evaporation. The fission is regarded as a collective process of the nucleus. After the emission of a few fast particles, the residual nucleus attains an equilibrated state, where fission competes with the particle evaporation. A large number of intermediate nuclei each with its own fission characteristics are formed. Therefore, small deviations in the cascade step may lead to a very different population of the intermediate nucleus, which contributes differently to the mass yield distribution.

\section{Summary}

The recoil properties of 137 radionuclides formed in the photospallation reactions of ${ }^{27} \mathrm{Al},{ }^{\text {nat }} \mathrm{V},{ }^{\text {nat }} \mathrm{Cu},{ }^{93} \mathrm{Nb},{ }^{\text {nat }} \mathrm{Ag}$, ${ }^{\text {nat }} \mathrm{Ta}$, and ${ }^{197} \mathrm{Au}$, induced by bremsstrahlung of end-point energies of $E_{0}=600-1100 \mathrm{MeV}$, have been investigated by the thicktarget thick-catcher method. Reflecting the resonance character in photonuclear reactions, the mean ranges in the forward and backward directions are $E_{0}$-independent at the studied energies and exhibit two components of the $(\gamma, x$ nyp) $(x, y \geq 1)$ and the $(\gamma, x \mathrm{n})(x \geq 1)$ reactions. The forward-to-backward ratios $F / B$ were found to be independent of the mass difference $\Delta A$ between a product $\left(A_{\mathrm{p}}\right)$ and a target $\left(A_{\mathrm{t}}\right)$ and also of $A_{\mathrm{t}}$. The kinematic properties of the product nuclei were analyzed by the two-step vector velocity model. The forward velocity $v$ after the first step of photon reaction is quite different from that of proton reaction at $E_{\mathrm{p}} \leq 3 \mathrm{GeV}$, though the difference disappears at higher energies. On the other hand, the mean kinetic energy $T$ of the residual nucleus in the second step is almost equal to that of proton reaction irrespective of $E_{\mathrm{p}}$. The PICA3/GEM code at $E_{0}=600-1100 \mathrm{MeV}$ reproduces the experimental results of ${ }^{\text {nat }} \mathrm{V},{ }^{\text {nat }} \mathrm{Cu},{ }^{93} \mathrm{Nb}$, and ${ }^{\text {nat }} \mathrm{Ag}$ fairly well, but the same calculation for heavier targets gives $T$ values lower than the experimental results, indicating some nuclearstructure effect such as a medium effect notably at $A_{\mathrm{t}} \geq 100$. The average kinetic energy of the emitted particles in a random-walk process of both the photon and proton reactions, $\varepsilon_{\mathrm{s}}=T /\left(\Delta A / A_{\mathrm{t}}\right)$, was found to increase along with an increase of $A_{\mathrm{t}}$ and to become constant above $A_{\mathrm{t}}=100$. The $\varepsilon_{\mathrm{s}}$ values of photon reaction appear to be slightly lower than those of proton reactions for ${ }^{\text {nat }} \mathrm{Ag}$, nat $\mathrm{Ta}$, and ${ }^{197} \mathrm{Au}$. This difference may indicate the lower excitation energy left after the first step in photon reactions than that in proton reactions, which is not apparent in $T$.

The recoil properties and yields of radionuclides formed in the photofission of ${ }^{197} \mathrm{Au}$ and ${ }^{209} \mathrm{Bi}$ were obtained at $E_{0}=$ $300-1100 \mathrm{MeV}$ using the catcher foil technique with the aid of intensive chemical separation. Both the $F / B$ and $T$ values of the fission products of ${ }^{197} \mathrm{Au}$ were clearly distinguished from those of the photospallation products. The variation of $F / B$ and $T / E_{\mathrm{C}}$ for ${ }^{24} \mathrm{Na}$ produced from ${ }^{27} \mathrm{Al},{ }^{\text {nat }} \mathrm{V},{ }^{\text {nat }} \mathrm{Cu}$, ${ }^{\text {nat }} \mathrm{Ag}$, and ${ }^{197} \mathrm{Au}$ showed a change in the production mechanism from spallation to fragmentation with respect to $A_{\mathrm{t}}$. The charge- and mass-distributions of the photofission of ${ }^{197} \mathrm{Au}$ and ${ }^{209} \mathrm{Bi}$ were successfully deduced based on the 58 and 63 fission yields, respectively. The charge distribution was reproduced by a Gaussian function with the most probable charge $Z_{\mathrm{p}}$ expressed by a linear function of the product mass number $A$, i.e., $Z_{\mathrm{p}}=$ $R A+S$, and with the $A$-independent width $F W H M_{\mathrm{CD}}$. The charge distribution parameters $R, S$, and $F W H M_{\mathrm{CD}}$ were found to be independent of $E_{0}$ above $600 \mathrm{MeV}$, reflecting the resonance nature in photonuclear reactions at intermediate energies. The numbers of pre- and post-fission neutrons were deduced to be $v_{\text {pre }}=11 \pm 1$ and $v_{\text {post }}=1.7 \pm 0.3$ for ${ }^{197} \mathrm{Au}$, and to be $v_{\text {pre }}=12 \pm 1$ and $v_{\text {post }}=1.4 \pm 0.3$ for ${ }^{209} \mathrm{Bi}$, assuming the unchanged charge distribution hypothesis. Based on the charge distribution parameters, the symmetric mass yield distribution with the most probable mass $A_{\mathrm{p}}$ of 92 m.u. and the width $F W H M_{\mathrm{MD}}$ of 39 m.u. was obtained for ${ }^{197} \mathrm{Au}$ at $E_{0} \geq 600$ 
$\mathrm{MeV}$. The $A_{\mathrm{p}}$ value for ${ }^{209} \mathrm{Bi}$ was larger by $4 \mathrm{~m} . \mathrm{u}$. than that for ${ }^{197} \mathrm{Au}$ and the $F W H M_{\mathrm{MD}}$ was smaller by 6 m.u. The comparisons of the charge- and mass-distribution parameters with the latest calculations using the PICA3/GEM code indicated some defects in the nuclear and reaction models on which the code was based.

Acknowledgement. The main part of the present study was done while the author belonged to Radiochemistry Laboratory, Department of Chemistry, Faculty of Science, Kanazawa University. The author would like to express his gratitude to Prof. K. Sakamoto of Department of Chemistry, Faculty of Science, Kanazawa University, Prof. I. Fujiwara of Department of Economics, Faculty of Economics, OtemonGakuin University, Prof. S. Shibata of Research Reactor Institute, Kyoto University, Prof. M. Furukawa of Faculty of Environmental and Information Sciences, Yokkaichi University, and Dr. Oura of Department of Chemistry, Graduate School of Science, Tokyo Metropolitan University, for their guidances and advices throughout the course of this work. The students of the Radiochemistry Laboratory of Kanazawa University are thanked for their cooperation in radiochemical separations and radioactivity measurements. The author would like to acknowledge Prof. K. Masumoto and the ES crew members of the High Energy Accelerator Research Organization at Tanashi for their invaluable cooperations in the course of experiments.

\section{References}

(1) K. Sakamoto, M. Yoshida, Y. Kubota, T. Fukasawa, A. Kunugise, Y. Hamajima, S. Shibata, and I. Fujiwara, Nucl. Phys. A 501, 693 (1989).

(2) K. Sakamoto, Y. Hamajima, M. Soto, Y. Kubota, M. Yoshida, A. Kunugise, M. Masatani, S. Shibata, M. Imamura, M. Furukawa, and I. Fujiwara, Phys. Rev. C 42, 1545 (1990).

(3) Y. Oura, A. Yazawa, M. Yoshida, S. R. Sarkar, K. Sakamoto, S. Shibata, I. Fujiwara, and M. Furukawa, Radiochim. Acta 68, 27 (1995).

(4) K. Sakamoto, S. R. Sarkar, Y. Oura, H. Haba, H. Matsumura, Y. Miyamoto, S. Shibata, M. Furukawa, and I. Fujiwara, Phys. Rev. C 59, 1497 (1999).

(5) S. Shibata, M. Imamura, T. Miyachi, M. Mutou, K. Sakamoto, Y. Hamajima, M. Soto, Y. Kubota, M. Yoshida, and I. Fujiwara, Phys. Rev. C 35, 254 (1987).

(6) S. R. Sarkar, M. Soto, Y. Kubota, M. Yoshida, T. Fukasawa, K. Matsumoto, K. Kawaguchi, K. Sakamoto, S. Shibata, M. Furukawa, and I. Fujiwara, Radiochim. Acta 55, 113 (1991).

(7) S. R. Sarkar, Y. Kubota, T. Fukasawa, K. Kawaguchi, K. Sakamoto, S. Shibata, and I. Fujiwara, Radiochim. Acta 55, 139 (1991).

(8) S. R. Sarkar, Y. Oura, K. Kawaguchi, A. Yazawa, K. Sakamoto, S. Shibata, and I. Fujiwara, Radiochim. Acta 62, 7 (1993).

(9) S. Shibata, M. Imamura, K. Sakamoto, S. Okizaki, S. Shibutani, H. Matsumura, M. Furukawa, I. Fujiwara, H. Nagai, and K. Kobayashi, Radiochim. Acta 80, 181 (1998).

(10) H. Matsumura, K. Washiyama, H. Haba, Y. Miyamoto, Y. Oura, K. Sakamoto, S. Shibata, M. Furukawa, I. Fujiwara, H. Nagai, T. Kobayashi, and K. Kobayashi, Radiochim. Acta 88, 313 (2000).

(11) B. G. Harvey, Annu. Rev. Nucl. Sci. 10, 235 (1960).

(12) J. M. Alexander, Nuclear Chemistry, edited by L. Yaffe (Academic, New York 1968), Vol. I, p. 273.

(13)L. Winsberg, Phys. Rev. C 22, 2116 (1980) and C 22, 2123 (1980).
(14) A. Järund and B. Forkman, Z. Physik A 281, 47 (1977).

(15) A. A. Arakelyan, A. R. Balabekyan, A. S. Danagulyan, and A. G. Khudaverdyan, Nucl. Phys. A 534, 535 (1991).

(16) K. A. Amroyan, S. A. Barsegyan, and N. A. Demekhina, Phys. At. Nucl. 56, 6 (1993).

(17) K. A. Amroyan, S. A. Barsegyan, and N. A. Demekhina, Phys. At. Nucl. 56, 712 (1993).

(18) H. Haba, H. Matsumura, Y. Miyamoto, K. Sakamoto, Y. Oura, S. Shibata, M. Furukawa, and I. Fujiwara, J. Radioanal. Nucl. Chem. 239, 133 (1999).

(19)I. Fujiwara, H. Haba, H. Matsumura, Y. Miyamoto, K. Sakamoto, Y. Oura, S. Shibata, and M. Furukawa, Czech. J. Phys. 49, 831 (1999).

(20) H. Haba, H. Matsumura, K. Sakamoto, Y. Oura, S. Shibata, M. Furukawa, and I. Fujiwara, Radiochim. Acta 85, 1 (1999).

(21)H. Haba, H. Matsumura, K. Sakamoto, Y. Oura, S. Shibata, M. Furukawa, and I. Fujiwara, Radiochim. Acta 88, 375 (2000).

(22) M. C. Duijvestijn, A. J. Koning, J. P. M. Beijers, A. Ferrari, M. Gastal, J. van Klinken, and R. W. Ostendorf, Phys. Rev. C 59, 776 (1999).

(23)R. E. Prael and H. Lichtenstein, Los Alamos National Laboratory Report No. LA-UR-89-3014 (1989).

(24)H. Haba, M. Igarashi, K. Washiyama, H. Matsumura, M. Yamashita, K. Sakamoto, Y. Oura, S. Shibata, M. Furukawa, and I. Fujiwara, J. Nucl. Radiochem. Sci. 1, 53 (2000).

(25)H. Haba, M. Igarashi, K. Washiyama, H. Matsumura, M. Yamashita, K. Sakamoto, Y. Oura, S. Shibata, M. Furukawa, and I. Fujiwara, J. Nucl. Radiochem. Sci. 1, 69 (2000).

(26) H. Haba, M. Kasaoka, M. Igarashi, K. Washiyama, H. Matsumura, Y. Oura, S. Shibata, K. Sakamoto, M. Furukawa, and I. Fujiwara, Radiochim. Acta 90, 371 (2002)

(27)T. A. Gabriel and R. G. Alsmiller, Jr., Phys. Rev. 182, 1035 (1969).

(28) T. A. Gabriel, M. P. Guthrie, and O. W. Hermann, Oak Ridge National Laboratory Report No. ORNL-4687 (1971).

(29)C. Y. Fu, PICA95, An Intra-Nuclear Cascade Code for 25-MeV to 3.5-GeV Photon-Induced Nuclear Reactions, presented at SATIF-3, Sendai, May 12-13, 1997.

(30) T. Sato, K. Shin, S. Ban, Y. Namito, H. Nakamura, and H. Hirayama, Nucl. Instr. Meth. A 437, 471 (1999).

(31)T. Sato, K. Shin, S. Ban, T. A. Gabriel, C. Y. Fu, and H. S. Lee, Proc. of Advanced Monte Carlo on Radiation Physics, Particle Transport Simulation and Applications, Lisbon, Oct. 23-26, 2000 (private communication).

(32) S. Furihata, Nucl. Instr. Meth. B 171, 251 (2000).

(33) H. Haba, K. Sakamoto, H. Matsumura, Y. Oura, S. Shibata, M. Furukawa, and I. Fujiwara, Czech. J. Phys. (in press).

(34)H. Haba, K. Sakamoto, M. Igarashi, M. Kasaoka, K. Washiyama, H. Matsumura, Y. Oura, S. Shibata, M. Furukawa, and I. Fujiwara, Czech. J. Phys. (in press).

(35) K. Osada, T. Fukasawa, K. Kobayashi, Y. Hamajima, K. Sakamoto, S. Shibata, and I. Fujiwara, Res. Rept. Lab. Nucl. Sci. Tohoku Univ. 20, 299 (1987).

(36) H. Haba, Ph. D. Thesis (Kanazawa Univ. 1999).

(37) H. W. Bertini, Phys. Rev. 131, 1801 (1963).

(38)F. Atchison, Kernforschungsanlage Jülich GmbH Report No. Jül-Conf-34 (1980).

(39) G. Rudstam, Z. Naturforsch. 21a, 1027 (1966).

(40) L. Winsberg, Nucl. Instr. Meth. 150, 465 (1978).

(41) J. F. Ziegler, J. P. Biersack, and U. Littmark, The Stopping and Range of Ions in Solid (Pergamon, New York, 1985).

(42) M. Lagarde-Simonoff, S. Regnier, H. Sauvageon, and G. 
N. Simonoff, Nucl. Phys. A 260, 369 (1976).

(43) V. P. Crespo, J. M. Alexander, and E. K. Hyde, Phys. Rev. 131, 1765 (1963).

(44) N. T. Porile and S. Tanaka, Phys. Rev. 135, B122 (1964).

(45) J. C. Cumming, P. E. Haustein, and H. -C. Hseuh, Phys. Rev. C 18, 1372 (1978).

(46) J. B. Cumming, P. E. Haustein, and H. -C. Hseuh, Phys. Rev. C 24, 2162 (1981).

(47) G. D. Cole and N. T. Porile, Phys. Rev. C 25, 244 (1982).

(48) S. C. Fung and I. Perlman, Phys. Rev. 87, 623 (1952).

(49) R. Wolfgang and G. Friedlander, Phys. Rev. 94, 775 (1954).

(50) V. L. Volkova and F. P. Denisov, Sov. Phys. JETP 8, 372 (1959).

(51) A. M. Poskanzer, J. B. Cumming, and R. Wolfgang, Phys. Rev. 129, 374 (1963).

(52) B. Neidhart and K. Bächmann, J. Inorg. Nucl. Chem. 34,
423 (1972).

(53) J. B. Cumming and K. Bächmann, Phys. Rev. C 6, 1362 (1972).

(54)E. P. Steinberg and L. Winsberg, Phys. Rev. C 10, 1925 (1974).

(55) S. B. Kaufman, E. P. Steinberg, and M. W. Weisfield, Phys. Rev. C 18, 1349 (1978).

(56) C. F. Wang, G. D. Cole, and N. T. Porile, Phys. Rev. C 29, 569 (1984).

(57)C. F. Wang and N. T. Porile, Nucl. Phys. A 468, 711 (1987).

(58)E. Ross and K. Bächmann, Radiochim. Acta, 21, 13 (1974).

(59) A. P. Komar, B. A. Bochagov, A. A. Kotov, Yu. N. Ranyuk, G. G. Semenchuk, G. E. Solyakin, and P. V. Sorokin, Sov. J. Nucl. Phys. 10, 30 (1970). 